\title{
Stigmasterol, a Soy Lipid-Derived Phytosterol, Is an Antagonist of the Bile Acid Nuclear Receptor FXR
}

\author{
BETH A. CARTER, OLGA A. TAYLOR, DANIEL R. PRENDERGAST, TRACY L. ZIMMERMAN, \\ RICHARD VON FURSTENBERG, DAVID D. MOORE, AND SAUL J. KARPEN
}

\author{
Department of Pediatrics [B.A.C., D.R.P., O.A.T., T.L.Z., R.F., S.J.K.], Division of Pediatric Gastroenterology, Hepatology, and Nutrition, \\ Texas Children's Liver Center; Department of Molecular and Cellular Biology [D.D.M.], Baylor College of Medicine, Houston, \\ Texas 77030
}

\begin{abstract}
Phytosterols, components of soy-derived lipids, are among the proposed exacerbants of parenteral nutrition-associated cholestasis (PNAC). We investigated whether phytosterols contribute to bile acid (BA)-induced hepatocyte damage by antagonizing a nuclear receptor (NR) critically involved in hepatoprotection from cholestasis, FXR (farnesoid X receptor, NR1H4). In HepG2 cells, stigmasterol acetate (StigAc), a water-soluble Stig derivative, suppressed ligand-activated expression of FXR target genes involved in adaptation to cholestasis (i.e. BSEP, $F G F-19, O S T \alpha / \beta$ ). Furthermore, StigAc antagonized BA-activated, FXR target genes SHP and BSEP in FXR $+/+$, but not in FXR $-/-$ mouse hepatocytes. Both Stig and StigAc inhibited BA-activated, FXR-dependent reporter gene expression in transfected HepG2 cells, whereas the most prevalent phytosterol in lipids, $\beta$-sitosterol, had no inhibitory effect. Finally, among six ligand-activated NR-ligand binding domains (LBDs) tested, antagonism by StigAc was specific to only two (FXR and PXR, pregnane $X$ receptor, NR1I2). We demonstrate that Stig, a phytosterol prevalent in soy-derived PN lipid solutions, is a potent in vitro antagonist of the NR for bile acids FXR. (Pediatr Res 62: 301-306, 2007)
\end{abstract}

$\mathrm{S}$ ince its introduction, $\mathrm{PN}$ has revolutionized the care of neonates. Elevated transaminases and bilirubin levels occur often in infants on PN, but with short-term administration, resolution of liver damage is typical. Alternatively, infants necessitating long-term PN are at severe risk of lifethreatening complications. These include cirrhosis, liver failure, sepsis, coagulopathy, and death. Those most susceptible to these morbidities are premature infants, those with short bowel syndrome, and those with repeated bouts of sepsis (1). The incidence of PNAC may be as high as 50\% in infants who have received PN for as little as $2 \mathrm{~m}$ (1). The risk of developing end-stage liver disease is currently estimated at $20 \%-87 \%$ (1-3).

Most PN lipid emulsions are derived from soy and contain numerous phytochemicals, including sterols (phytosterols), structurally similar to both cholesterol and BAs (Fig. 1) (4,5).

Received August 28, 2006; accepted April 11, 2007.

Correspondence: Saul J. Karpen, M.D., Ph.D., Texas Children's Liver Center, Department of Pediatrics/GI, Hepatology \& Nutrition, Baylor College of Medicine, One Baylor Plaza, Houston, TX 77030; e-mail: skarpen@bcm.tmc.edu

Supported in part by National Institutes of Health Grant K12HD41648 and the American Gastroenterological Association/Roche Research Scholar Award in Liver Disease (B.A.C.), DK56239 (S.J.K.), and the Texas Gulf Coast Digestive Disease Center (DK56338).
In humans, most $(>95 \%)$ dietary phytosterols are excreted in the feces due to the enterocyte apical ABCG5/G8 transporter, which acts as a gatekeeper to their systemic entry, (6) but is bypassed with i.v. administration and subsequently excreted into bile via ABCG5/G8 located on the hepatocyte canalicular membrane $(5,6)$.

There are three principal phytosterols in soy lipid: $\beta$-sitosterol, campesterol, and Stig. Several lines of evidence support a potential role for phytosterols as contributors to the development of PNAC: (1) children with PNAC have markedly elevated levels of phytosterols such as Stig in their serum, ranging above $1 \mathrm{mM}$ (normal $=36 \mu \mathrm{M}$ ); (2) a reduction in $\mathrm{PN}$ lipid administration results in a marked reduction in both serum phytosterol and bilirubin levels; (3) i.v. administration of phytosterols in neonatal piglets leads to an increase in serum BAs and reduced BA-dependent bile flow; and (4) newborn piglets provided fish oil-derived lipids i.v. maintain normal bile flow and transaminase levels, whereas piglets given soybean-derived lipids develop cholestasis and impaired bile flow rates (7-9).

Multiple members of the NR superfamily play central roles in the adaptive response to cholestasis, but FXR, NR1H4 has emerged the central BA "sensor" that plays a critical role in maintaining safe intrahepatic BA levels (10). BA-activated FXR mediates hepatoprotection from excess BAs by coordinating a transcriptional reprogramming of hepatocytes: (1) reducing sinusoidal BA import via suppression of $\mathrm{Na}^{+}$taurocholate cotransporter polypeptide (NTCP), SLC10A1; (2) reducing BA synthesis via repression of CYP7A1; and (3) increasing intrahepatic BA efflux across canalicular and sinusoidal membranes via up-regulation of bile salt export pump (BSEP) ABCB11 and organic solute transporter (OST) $\alpha / \beta$ genes, respectively (10-15). Gene knockout studies demonstrate that FXR $-/-$ mice lack these hepatoprotective mechanisms and are ultrasensitive to BA-induced injury, and treat-

\footnotetext{
Abbreviations: BA, bile acid; BSEP, bile salt export pump; CDCA, chenodeoxycholic acid; FGF, fibroblast growth factor; FXR, farnesoid X receptor; LBD, ligand-binding domain; LXR, liver X receptor; NR, nuclear receptor; NTCP, sodium taurocholate cotransporting polypeptide; OST, organic solute transporter; PN (AC), parenteral nutrition (-associated cholestasis); PPAR $\gamma$, peroxisome proliferator-activated receptor gamma; PXR, pregnane $\mathrm{X}$ receptor; RXR, retinoid X receptor; $\mathbf{S H P}$, small heterodimer partner; Stig (Ac), stigmasterol (acetate); VDR, vitamin D receptor
} 

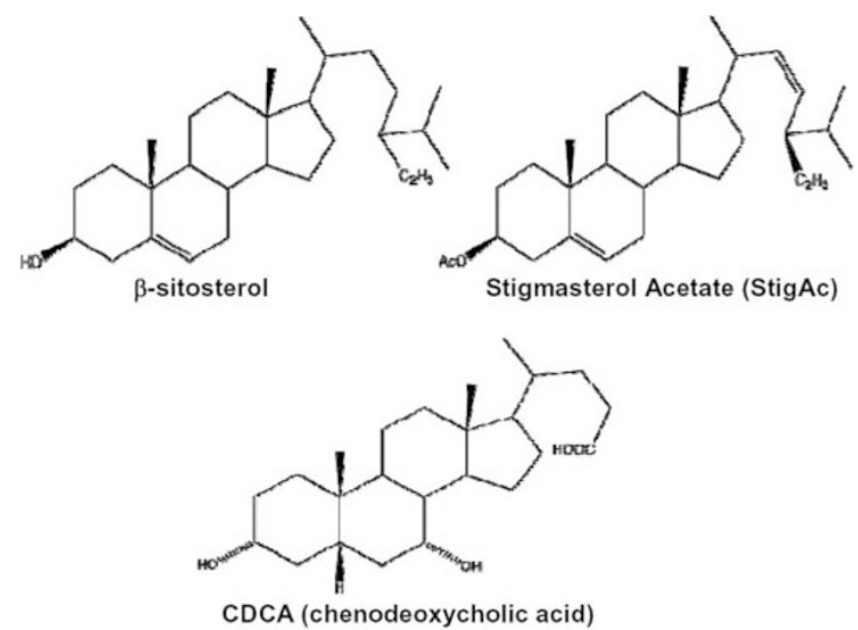

Figure 1. Structures of selected sterols. The nuclear ring structures of two phytosterol compounds ( $\beta$-sitosterol and StigAc) are illustrated. StigAc is a commercially available Stig derivative with an acetate group at position three of the Stig backbone. The structural similarity of these phytosterols to the primary bile acid, chenodeoxycholic acid (CDCA) is also illustrated.

ment of rats with FXR agonists protects against cholestasis $(16,17)$.

Although multiple hypotheses have been proposed to explain the pathogenesis of PNAC, a multifactorial model appears to be at play (18). Current thinking supports that prematurity and immature hepatic function, lack of enteral feedings, bacterial translocation (leading to recurrent sepsis), enrichment of the BA pool with toxic BAs, and the prolonged use of lipid emulsions are contributors (1-3). We report that Stig, a principal phytosterols present in soy-derived lipid infusates, is a potent antagonist of the critical NR BA sensor FXR and provides a novel molecular connection between nutritional components and regulation of essential liver gene expression.

\section{MATERIALS AND METHODS}

Tissue culture. HepG2 cells were obtained from American Type Tissue Culture (Manassas, VA). Cells for transfections were grown as monolayers to $75 \%$ confluence at $37^{\circ} \mathrm{C}$ in a humidified atmosphere of $95 \%$ air and $5 \% \mathrm{CO}_{2}$ in $10 \mathrm{~mL}$ of medium that consisted of modified Eagle's medium (MEM) (GIBCO) supplemented with $10 \%$ fetal bovine serum (FBS), $1 \%$ penicillinstreptomycin, and $1 \%$ L-glutamine. Transfection treatment medium was identically prepared with the exception that $10 \%$ FBS was replaced by $10 \%$ charcoal-stripped medium. Cells were subcultured on a routine basis per standard protocol.

Primary mouse hepatocytes were isolated via a standardized perfusion protocol and plated in complete William's E medium in six-well dishes at a density of $250,000 / \mathrm{mL}$ (19). For quantitative reverse-transcriptase polymerase chain reaction (qRTPCR), mouse hepatocytes were seeded for $3 \mathrm{~h}$ in complete William's E medium supplemented with penicillin/streptomycin, glutamine/ gentamicin, insulin-transferrin-sodium selenite, glucagon, 10\% FBS, and amphotericin B, followed by $24-\mathrm{h}$ treatments in $0.25 \%$ charcoal-stripped William's E medium. For qRTPCR, HepG2 cells were seeded at $500,000 / \mathrm{mL}$ for $24 \mathrm{~h}$ in complete Dulbecco's modified Eagle's medium (DMEM) (GIBCO) supplemented with penicillin/streptomycin, L-glutamine, and 10\% FBS followed by $24-\mathrm{h}$ treatments in $0.25 \%$ charcoal-stripped DMEM.

Treatments. Phytosterols and derivatives were obtained from Steraloids, Inc. (Newport, RI). StigAc (Fig. 1) has superior solubility and stability compared with its parent compound, allowing for an alternative, more standardized phytosterol treatment protocol and hence its use in the majority of our experiments. Phytosterol treatment medium for transfections was prepared by mixing a $20-\mathrm{mM}$ stock solution of the phytosterol in $10 \%$ charcoalstripped MEM medium. This mixture was vortexed and sonicated (Ultrasonic,
Inc.) for $48 \mathrm{~h}$, then syringe-filtered $(0.4 \mu \mathrm{m})$ into aliquots, and refiltered into a sterile $0.2-\mu \mathrm{m}$ vacuum flask producing a transparent solution of phytosterol in medium. The precise micromolar quantity of phytosterol in each treatment solution was then determined by gas chromatography mass spectrometry (GCMS) (courtesy of Dr. William E. O'Brien, Department of Genetics, Baylor College of Medicine) (20). StigAc treatment medium for qRTPCR experiments was similarly prepared in $0.25 \%$ charcoal-stripped William's E (primary mouse hepatocytes) or DMEM (HepG2 cells) with subsequent validation of StigAc concentrations in the medium via GCMS (20). HepG2 cell viability, as measured by $\mathrm{EtBr}$ exclusion, after $24 \mathrm{~h}$ of incubation, was unaffected by Stig: vehicle (75\%), $10 \mu \mathrm{M}$ Stig (75\%), CDCA $50 \mu \mathrm{M}(88 \%)$, or CDCA + Stig $(92 \%)$.

NR ligands were obtained from Sigma Chemical Co., except for the LG100268, which was generously provided by Ligand Pharmaceuticals, Inc. (San Diego, CA). In most cases, these ligands were solubilized in dimethyl sulfoxide before cell treatments (4-pregnen-3 $\beta$-ol-20-one carbonitrile, $22-\mathrm{R}$ hydroxycholesterol, rosiglitazone, LG100268, vitamin D). In the case of CDCA, the sodium salt of this ligand was solubilized in sterile water.

Transfections. HepG2 cells were seeded at a density of 250,000 cells/well in 12-well dishes and transfected with Fugene (Roche) transfection reagent; $0.75 \mu \mathrm{g}$ of the indicated luciferase reporter plasmids were cotransfected with $0.1 \mathrm{~g} \mathrm{hFXR}, 0.1 \mu \mathrm{g}$ retinoic X receptor (RXR), $\mathrm{hRXR} \alpha$, and $0.05 \mu \mathrm{g}$ of pRSVrenilla to monitor for transfection efficiency. For transfections testing phytosterol effect on full-length FXR (Figs. 2 and 3A), HepG2 cells were cotransfected with a luciferase reporter plasmid (pECRELuc) containing five copies of an RXR:FXR binding site and expression vectors for hFXR and $\mathrm{hRXR} \alpha$, along with a pRSVrenilla internal control. Cells were then treated with $0-100 \mu \mathrm{M}$ CDCA and $0-10 \mu \mathrm{M}$ of various phytosterols. After $24 \mathrm{~h}$, cells were harvested and processed on a luminometer (Luminoscan Ascent), and results normalized to RSVrenilla activity to control for transfection efficiency. Transfections were performed at least six times in triplicate. HepG2 cells were cotransfected with a Gal4 luciferase reporter containing five copies of a Gal4 binding site, an expression plasmid vector for a Gal4-DNAbinding domain NR, ligand-binding domain (LBD) fusion protein, an RXR $\alpha$ expression vector, and a pRSVrenilla internal control (21). Cells were treated with agonist or agonist plus $10 \mu \mathrm{M}$ StigAc and $24 \mathrm{~h}$ later harvested and processed, and luciferase activities normalized as before. The agonist ligands used were FXR, CDCA (100 $\mu \mathrm{M})$; pregnane X receptor (PXR), 4-pregnen$3 \beta$-ol-20-one- $16 \alpha$-carbonitrile (PCN, $10 \mu \mathrm{M}$ ); vitamin D receptor (VDR), $1 \alpha$, 25-dihydroxyvitamin $\mathrm{D}_{3}(100 \mathrm{nM})$; liver $\mathrm{X}$ receptor (LXR) $\alpha$, 22-(R)hydroxycholesterol $(10 \mu \mathrm{M})$; RXR $\alpha$, LG100268 (1 $\mu \mathrm{M})$; peroxisome proliferator-activated receptor $\gamma(\operatorname{PPAR} \gamma)$, rosiglitazone $(1 \mu \mathrm{M})$.

qRTPCR. Primary mouse hepatocytes were cultured as noted above and treated for $24 \mathrm{~h}$ with vehicle alone $(0.25 \%$ charcoal-stripped William's E medium) or $100 \mu \mathrm{M}$ CDCA with or without $10 \mu \mathrm{M}$ StigAc, and HepG2 cells

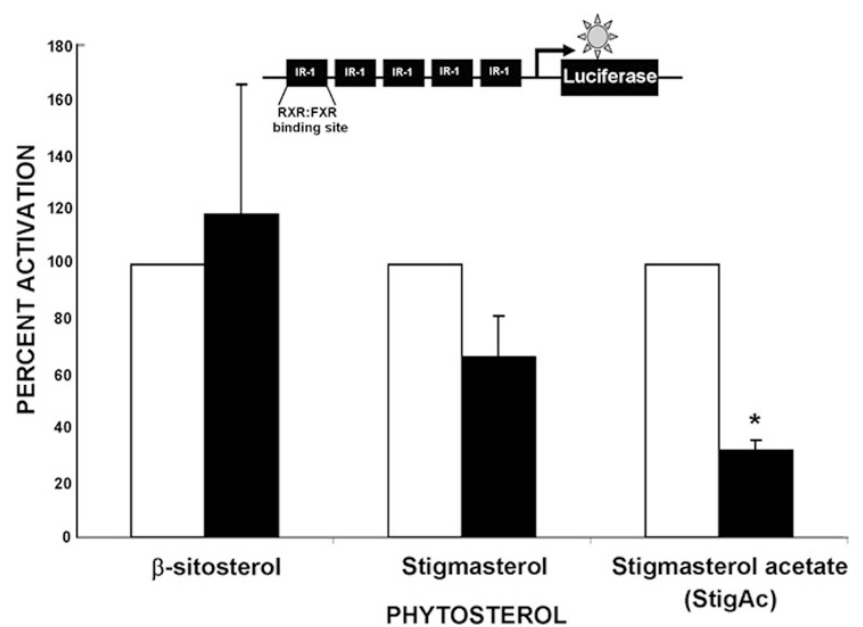

Figure 2. Stig and StigAc suppress FXR-mediated gene expression in vitro; $\beta$-sitosterol does not. A luciferase reporter/FXR response plasmid (pECRELuc) was transfected into HepG2 cells along with FXR and RXR $\alpha$ expression plasmids and then treated with $100 \mu \mathrm{M}$ CDCA and $10 \mu \mathrm{M}$ of phytosterols. Cells were harvested $24 \mathrm{~h}$ later, and results normalized to cotransfected pRSVrenilla. Results in the presence of CDCA are presented as the percentage of activation relative to normalized luciferase expression in the presence of agonist (100\%). *p $<0.05$ vs CDCA alone. $\square$, CDCA + vehicle; CDCA + phytosterol. 

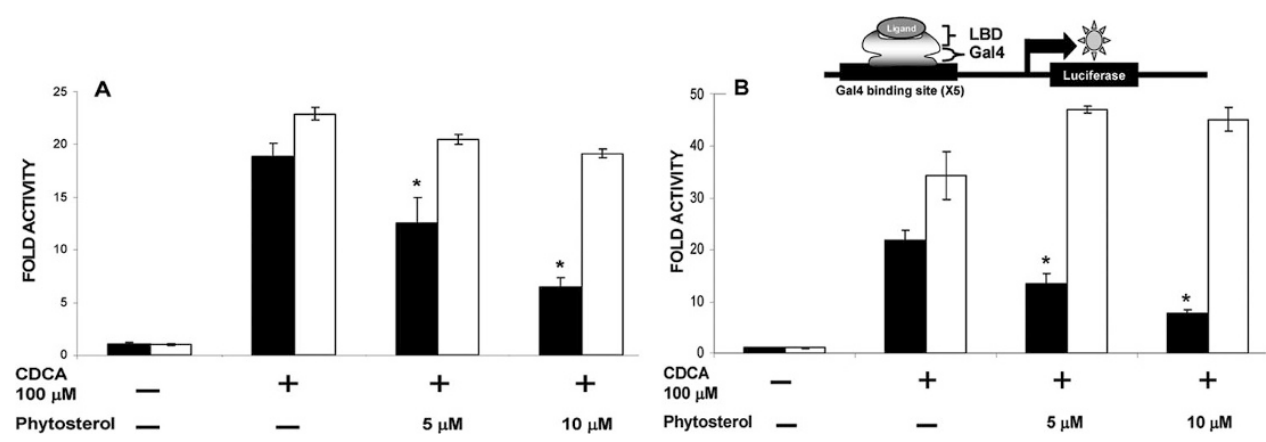

Figure 3. StigAc is a potent in vitro antagonist of full-length FXR and FXR-LBD. (A) Inhibition of full-length FXR by StigAc. A luciferase reporter plasmid containing five copies of a full-length RXR:FXR binding site (pECRELuc) was transfected into HepG2 cells along with FXR and RXR expression plasmids, followed by treatment with $100 \mu \mathrm{M}$ CDCA and StigAc $(0-10 \mu \mathrm{M})$. (B) Inhibition of Gal4-FXR-LBD by StigAc. A luciferase reporter plasmid (GSTKLuc) was transfected into HepG2 cells along with an FXR-LBD construct plasmid and RXR $\alpha{ }^{*} p<0.05$ s CDCA alone. $\square$, SticAg; $\square, \beta$-sitosterol.

were plated for $24 \mathrm{~h}$ and then treated for $24 \mathrm{~h}$ with vehicle alone $(0.25 \%$ charcoal-stripped DMEM medium) or 50-100 $\mu \mathrm{M}$ CDCA with or without 10 $\mu \mathrm{M}$ StigAc. Total RNA was isolated using the RNAeasy kit (Qiagen), cDNA was synthesized from 7.5 or $10 \mu \mathrm{g}$ of total RNA using the StrataScript First-Strand synthesis system (Stratagene, La Jolla, CA) and used to determine relative small heterodimer partner (SHP), BSEP, fibroblast growth factor (FGF), FGF-19, OST $\alpha$, and OST $\beta$ mRNA expression using an ABI PRISM 7700 Sequence Detection System instrument and software (Applied Biosystems, Inc., Foster City, CA) as reported previously (34). Quantitative expression values were extrapolated from standard curves and normalized to a cyclophilin or $18 \mathrm{~S}$ standard.

\section{RESULTS}

Initial transfections were designed to determine which, if any, of the phytosterols present in soy-derived lipid infusates affected FXR activity by the potent BA ligand CDCA. Stig and StigAc suppress BA-activated, FXR-mediated reporter gene expression (Fig. 2). $\beta$-Sitosterol, the most prevalent phytosterol in soy-derived lipids, has no significant inhibitory effect on CDCA-activated, FXR-mediated reporter gene expression, whereas campesterol has minimal and insignificant inhibition (data not shown). Phytosterol treatments alone had no significant effect on FXR reporter gene activation (data not shown).

Because Stig emerged as the most potent FXR antagonist among the three main phytosterols, we next tested the in vitro dose-response effect of StigAc on the function of ligandactivated full-length FXR (Fig. 3A). Increasing concentrations of StigAc $(0-10 \mu \mathrm{M})$ suppress CDCA-activated FXR with an $\mathrm{IC}_{50}$ of $5-10 \mu \mathrm{M}$, which is well below the physiologic range of Stig levels found in the serum of patients with serological evidence of PNAC (9). $\beta$-Sitosterol, once again, does not significantly antagonize CDCA-activated, full-length FXR reporter gene expression. Transfections in CV-1 cells similarly demonstrate that StigAc antagonizes BA-activated full-length FXR in this FXR-null cell line (data not shown).

Whether StigAc-mediated antagonism required the FXRLBD was addressed with Gal4-FXR-LBD transfections in HepG2 cells (Fig. 3B). StigAc antagonizes CDCA activation of a Gal4-FXR-LBD in a dose-dependent fashion similar to that seen with full-length FXR $(65 \%$ at $10 \mu \mathrm{M})$ (compare Fig. $3 A$ with $3 B$ ). In contrast, $\beta$-sitosterol does not inhibit CDCA-activated Gal4-FXR-LBD. Such results suggest that the inhibition of CDCA-activated FXR by StigAc involves competition at the FXR-LBD. Again, this suppression of
CDCA-activated FXR-LBD by StigAc is recapitulated in transfected CV-1 (FXR-null) cells, and StigAc had no significant effect on reporter gene expression (data not shown).

Given the structure of phytosterols and the multiple NRs involved in BA homeostasis, it was important to determine whether StigAc inhibited ligand activation of other liverenriched NRs (Fig. 4). The specificity of StigAc effect was tested using GAL4-LBD constructs of FXR and five other members of the NR superfamily involved in BA metabolism and inflammation: $\mathrm{PXR}, \mathrm{LXR} \alpha, \operatorname{PPAR} \gamma$, retinoid $\mathrm{X}$ receptor $\alpha$ $(\mathrm{RXR} \alpha)$, and VDR. In addition to suppressing CDCAactivated FXR-LBD activity, StigAc also suppresses ligandactivated PXR-LBD activity (Fig. 4). Except for a slight increase in $\operatorname{RXR} \alpha$ LBD activity, StigAc has no discernible effect on any other NR LBD tested.

Because StigAc significantly inhibited expression of FXRregulated plasmids transfected into HepG2 and CV-1 cells, we sought to determine whether it affected CDCA activation of native FXR-responsive genes (BSEP, FGF-19, and $O S T \alpha / \beta)$ in HepG2 cells (Fig. $5 A-D$ ). Each of these genes contains an FXR response element in its promoter region that is upregulated upon treatment with $\mathrm{CDCA}$, and their gene products

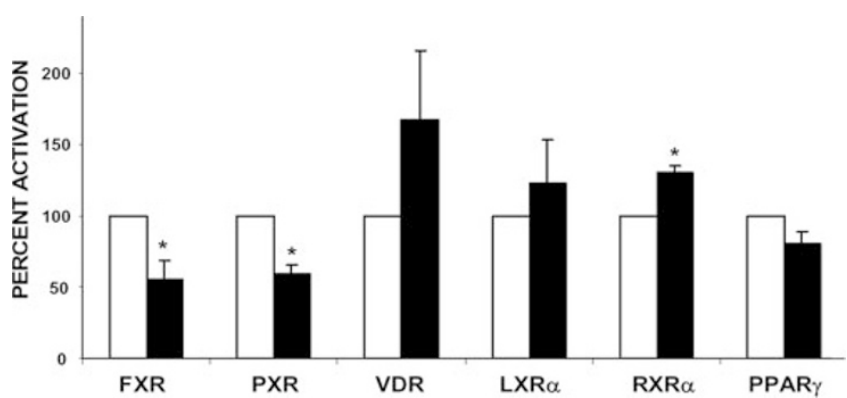

Figure 4. Specificity of StigAc effect on various NR LBDs. HepG2 cells were transfected with the GAL4 luciferase reporter, an RXR expression plasmid, and a series of chimeras in which the GAL4 DNA binding domain is fused to the indicated NR LBD. Cells were treated with the appropriate agonist ligand $(\square)$ or agonist plus StigAc $(10 \mu \mathrm{M}, \square)$. Results in the presence of StigAc are represented as the percent of activation relative to the normalized luciferase expression in the presence of agonist (100\%). The ligands used were FXR, CDCA $(100 \mu \mathrm{M})$; PXR, 4-pregnen-3 $\beta$-ol-20-one-16 $\alpha$-carbonitrile (PCN, $10 \mu \mathrm{M})$; VDR, $1 \alpha$,25-dihydroxyvitamin $\mathrm{D}_{3}$ (100 nM); LXR $\alpha$, 22-(R)hydroxycholesterol $(10 \mu \mathrm{M})$; RXR $\alpha$, LG100268 (1 $\mu \mathrm{M})$; PPAR $\gamma$, rosiglitazone $(1 \mu \mathrm{M}) . * p<0.05 v s$ ligand alone. 

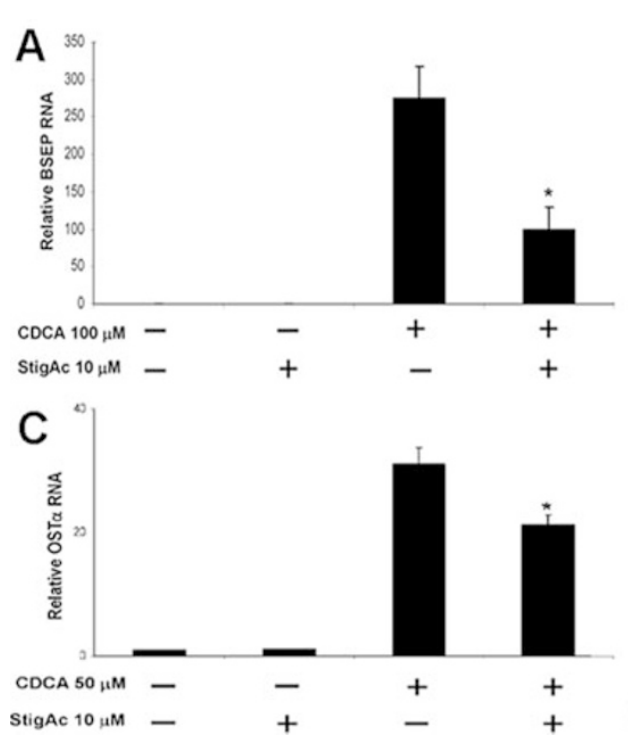

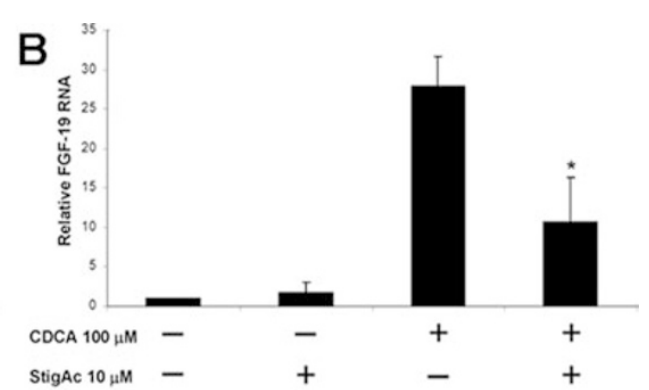

D

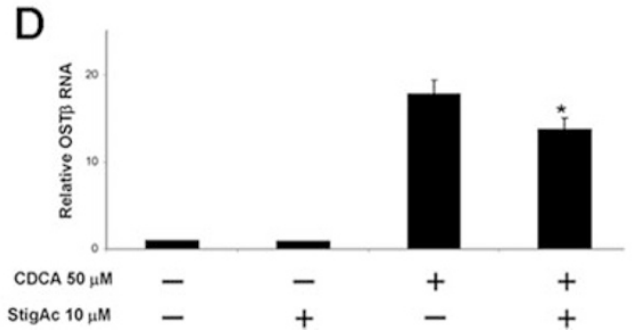

Figure 5. StigAc antagonizes $C D C A$ activation of FXR target genes $B S E P, F G F$ 19, OST $\alpha$, and $O S T \beta$ in HepG2 cells. Human hepatoblastoma (HepG2) cells were plated and then treated for $24 \mathrm{~h}$. Cells were then harvested, RNA purified, and qRTPCR performed to determine FXR target gene $[B S E P(A), F G F-19(B)$, OST $\alpha(C)$, $\operatorname{OST\beta }(D)]$ expression relative to an $18 \mathrm{~s}$ control. $* p<0.05$ vs CDCA alone. are involved in the hepatoprotective response to BA overload (21-23). Neither vehicle nor $10 \mu \mathrm{M}$ StigAc alone affects $B S E P$ RNA expression (Fig. 5A). As expected, treatment with CDCA, up-regulates BSEP RNA expression 275-fold compared with vehicle. Exposure to only $10 \mu \mathrm{M}$ of StigAc markedly suppresses CDCA-mediated activation of BSEP RNA (from 275 to $100 \times, 65 \%$ ), nearly identical to the results noted in transfection experiments (Figs. 2 and 3).

Similarly, FGF-19 RNA levels are unchanged from basal levels when treated with StigAc (Fig. 5B) and increase 25-fold in the presence of CDCA. StigAc potently suppresses BAactivated $F G F-19$ RNA expression by $60 \%$. StigAc also antagonizes two additional CDCA-activated, FXR-mediated, BA homeostatic genes in HepG2 cells: OST $\alpha$ and OST $\beta$ (25\%-30\% suppression, Fig. 5C and $D$ ). Taken together, these qRTPCR experiments indicate that StigAc markedly impairs CDCA activation of native FXR-responsive genes in a humanderived liver cell line, HepG2.

Finally, the effect of StigAc on the FXR target genes SHP and BSEP was studied in hepatocytes derived from $\mathrm{FXR}+/+$ and $\mathrm{FXR}-/-$ mice. As expected, CDCA activates native expression of mouse $S H P$ and BSEP RNA in FXR $+/+$, but not FXR $-/-$ hepatocytes, albeit at levels less than those seen in HepG2 cells (Fig. 6). StigAc has no effect on CDCAactivated SHP RNA expression in hepatocytes derived from FXR-/- mice; however, $10 \mu \mathrm{M}$ StigAc significantly suppresses CDCA-activated SHP expression in FXR+/+ hepatocytes. StigAc antagonizes CDCA-activated BSEP expression in FXR $+/+$, but not FXR $-/-$ hepatocytes (Fig. 6B), although this suppression does not reach statistical significance, likely due to interanimal variability in the expression of this gene. Taken together, Figures 5 and 6 and reveal that, in addition to its effect on FXR-regulated gene expression in HepG2 cells, StigAc also inhibits native CDCA-activated FXR target gene expression in cultured mouse hepatocytes.

\section{DISCUSSION}

Those most at risk of developing PNAC are premature infants and those with short bowel syndrome, but the actual causes, or mechanisms underlying susceptibility, are unknown (1-3). Because cholestasis usually resolves once infants are weaned off PN, a contribution from a cholestatic component in the PN solution has been proposed, with previous literature supporting that this agent may reside within lipids $(8,9,15,24)$. Herein, we demonstrate that among the phytosterols, which are components of soy-derived lipid infusates, Stig is an antagonist of FXR, an NR responsible for maintaining intracellular BA homeostasis.

Phytosterols accumulate to extremely high serum levels in a rare disease due to mutations in either one of the recently identified ABC (ATP-binding cassette) transporter genes $A B C G 5$ and $A B C G 8$, which have increased intestinal absorption and impaired biliary secretion of phytosterols (25-27). The clinical phenotype includes extensive xanthomas, accelerated atherosclerosis, and premature death due to sterol deposition in coronary arteries (28). Although liver disease is not a hallmark of sitosterolemia, a recent report describes a patient
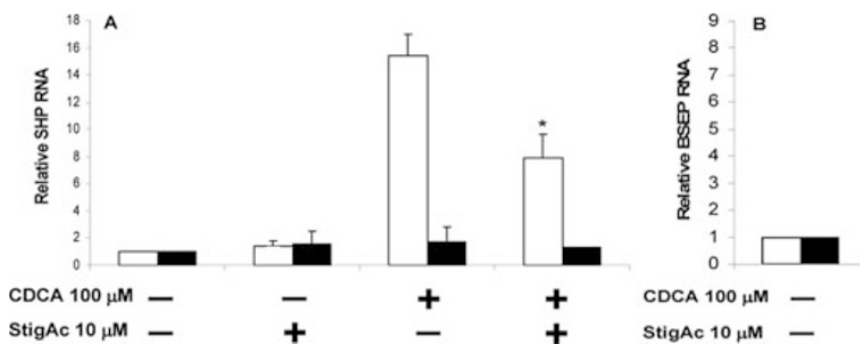

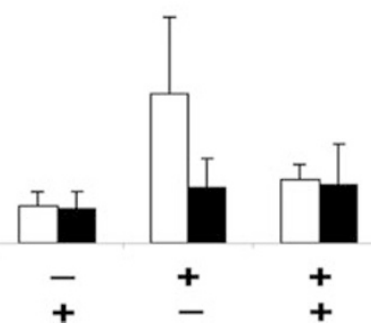

Figure 6. StigAc antagonizes CDCA activation of native FXR-responsive genes in hepatocytes derived from FXR $+/+$ mice, but not in FXR $-/-$ mice. Primary mouse were treated for $24 \mathrm{~h}$ as noted and then harvested, RNA was isolated, and qRTPCR was performed to determine FXR target gene [SHP $(A), B S E P(B)]$ mRNA expression compared with a cyclophilin control. ${ }^{*} p<0.01 v s$ CDCA alone. $\square$, FXR +/+;, FXR-/-. 
with cirrhosis requiring liver transplantation who was subsequently found to be a compound heterozygote $A B C G 8$ gene mutation (29). This patient also had serologic evidence of autoimmune hepatitis, suggesting that although sitosterolemia alone may not cause significant liver injury, a second insult to the liver in addition to hereditary phytosterolemia may trigger significant hepatocyte damage and cirrhosis. The apparent pathologic contradiction between the liver damage associated with $\mathrm{PN}$-associated phytosterolemia and the relatively benign liver effects in isolated hereditary sitosterolemia can be reconciled at several levels, mainly due to the differential clinical settings and route of exposure to phytosterols. First, PNAC is a disease of neonates, especially very small neonates, who have an obligate loading of soy lipids i.v., with the potential that the handling of phytosterols may be altered in these infants. Second, genes encoding BA transporter (i.e. BSEP) and BA conjugating/detoxification enzymes undergo postnatal development expression and extrapolating to premature infants suggest an inherently increased susceptibility to BA loads that resolves with age $(30,31)$. In the rat model, BSEP expression is low in fetal life and reaches adult levels by $4 \mathrm{wk}$ postnatally (30). The developmental expression of these transporters in humans has been studied to a lesser degree, but recent data suggest that there is a developmental increase in transporter RNA (i.e. BSEP, NTCP, and multidrug resistancerelated protein 2) (32). Third, unlike sitosterolemia, PNAC is a multifactorial disease that is exacerbated by ongoing systemic inflammation and infection, and the response of the liver in this setting is unknown. Previous investigators, including us, have established that lipopolysaccharides and cytokines alter the expression of crucial BA transport genes (e.g. NTCP and $B S E P$ ) in animal models and thereby directly contribute to cholestasis (33-35). It is quite possible that elevated levels of phytosterols "tip the balance," but this remains to be proven.

In human liver-derived HepG2 cells, StigAc antagonizes several BA-activated FXR target genes involved in restoring BA homeostasis: $S H P, B S E P, O s t \alpha / \beta$, and FGF-19. Mutations in $B S E P$ lead to intracellular BA accumulation and a serious hereditary cholestatic condition, progressive familial intrahepatic cholestasis type 2 (PFIC 2), whereas mutations in the other genes have yet to be described (36). The role of FGF-19 as a signaling molecule in cholestasis is interesting and evolving, but has been recently shown to be expressed in HepG2 cells $(22,37,39)$. There are likely to be species-dependent and variable roles played by FGF-15 (in mice) and FGF-19 (in humans) in cholestasis that have yet to be determined $(22,29,37-40)$.

In addition to FXR, our data reveal that StigAc inhibits the in vitro activity of ligand-activated PXR. Previous investigations in both wild-type and knockout animals have elucidated the integral role that PXR plays in "detoxifying" the liver of excess BAs (35). It is intriguing to speculate that StigAc may target genes regulated by PXR, which, in combination with its effects on FXR, would serve to doubly impair the ability of the liver to respond to BA loads.

In conclusion, this study provides evidence that Stig, a component of soy-derived lipids, has antagonist activity against a central NR involved in the adaptive response to
BA-mediated hepatotoxicity, FXR. These data suggest that Stig's antagonism of FXR target gene expression significantly compromises hepatoprotectant mechanisms that normally act to attenuate cholestasis (e.g. activation of BSEP, FGF-19, OST $\alpha / \beta$, and SHP). These in vitro data demonstrating Stig's role as an antagonist of the critical BA "sensor" FXR provides a basis for further exploration of the role of phytosterols in PNAC in appropriate animal models. It is only after the validation of these findings into in vivo models that we will be able to confirm what has been postulated for at least a decade: that a component of PN lipid infusates is a potential molecular exacerbant in the multifactorial pathogenesis of PNAC.

Acknowledgments. The authors gratefully appreciate the expertise at the Analyte Center at Baylor College of Medicine (Dr. William E. O'Brien, Ph.D., Director) for accurate measurements of phytosterol concentrations, and members of the Karpen Laboratory for their input into the design and execution of experiments.

\section{REFERENCES}

1. Kelly DA 1998 Liver complications of pediatric parenteral nutrition-epidemiology. Nutrition 14:153-157

2. Teitelbaum DH, Tracy T 2001 Parenteral nutrition-associated cholestasis. Semin Pediatr Surg 10:72-80

3. Btaiche IF, Khalidi N 2002 Parenteral nutrition-associated liver complications in children. Pharmacotherapy 22:188-211

4. Ostlund RE Jr 2002 Phytosterols in human nutrition. Annu Rev Nutr 22:533-549

5. Ostlund RE Jr, McGill JB, Zeng CM, Covey DF, Stearns J, Stenson WF, Spilburg CA 2002 Gastrointestinal absorption and plasma kinetics of soy Delta(5)phytosterols and phytostanols in humans. Am J Physiol Endocrinol Metab 282:E911-E916

6. Repa JJ, Berge KE, Pomajzl C, Richardson JA, Hobbs H, Mangelsdorf DJ 2002 Regulation of ATP-binding cassette sterol transporters ABCG5 and ABCG8 by the liver X receptors alpha and beta. J Biol Chem 277:18793-18800

7. Van Aerde JE, Duerksen DR, Gramlich L, Meddings JB, Chan G, Thomson AB, Clandinin MT 1999 Intravenous fish oil emulsion attenuates total parenteral nutrition-induced cholestasis in newborn piglets. Pediatr Res 45:202-208

8. Iyer KR, Spitz L, Clayton P 1998 BAPS prize lecture: New insight into mechanisms of parenteral nutrition-associated cholestasis: role of plant sterols. British Association of Paediatric Surgeons. J Pediatr Surg 33:1-6

9. Clayton PT, Bowron A, Mills KA, Massoud A, Casteels M, Milla PJ 1993 Phytosterolemia in children with parenteral nutrition-associated cholestatic liver disease. Gastroenterology 105:1806-1813

10. Wang H, Chen J, Hollister K, Sowers LC, Forman BM 1999 Endogenous bile acids are ligands for the nuclear receptor FXR/BAR. Mol Cell 3:543-553

11. Trauner M, Boyer JL 2003 Bile salt transporters: molecular characterization, function, and regulation. Physiol Rev 83:633-671

12. Boyer JL, Trauner M, Mennone A, Soroka CJ, Cai SY, Moustafa T, Zollner G, Lee JY, Ballatori N 2006 Upregulation of a basolateral FXR-dependent bile acid efflux transporter OSTalpha-OSTbeta in cholestasis in humans and rodents. Am J Physiol Gastrointest Liver Physiol 290:G1124-G1130

13. Goodwin B, Jones SA, Price RR, Watson MA, McKee DD, Moore LB, Galardi C, Wilson JG, Lewis MC, Roth ME, Maloney PR, Willson TM, Kliewer SA 2000 A regulatory cascade of the nuclear receptors FXR, SHP-1, and LRH-1 represses bile acid biosynthesis. Mol Cell 6:517-526

14. Karpen SJ 2002 Nuclear receptor regulation of hepatic function. J Hepatol 36:832850

15. Zollner G, Wagner M, Moustafa T, Fickert P, Silbert D, Gumhold J, Fuchsbichler A, Halilbasic E, Denk H, Marschall HU, Trauner M 2006 Coordinated induction of bile acid detoxification and alternative elimination in mice: role of FXR-regulated organic solute transporter-alpha/beta in the adaptive response to bile acids. Am J Physiol Gastrointest Liver Physiol 290:G923-G932

16. Sinal CJ, Tohkin M, Miyata M, Ward JM, Lambert G, Gonzalez FJ 2000 Targeted disruption of the nuclear receptor FXR/BAR impairs bile acid and lipid homeostasis. Cell 102:731-744

17. Liu Y, Binz J, Numerick MJ, Dennis S, Luo G, Desai B, MacKenzie KI, Mansfield TA, Kliewer SA, Goodwin B, Jones SA 2003 Hepatoprotection by the farnesoid X receptor agonist GW4064 in rat models of intra- and extrahepatic cholestasis. J Clin Invest 112:1678-1687

18. Sokol RJ 1997 Total parenteral nutrition-related liver disease. Zhonghua Min Guo Xiao Er Ke Yi Xue Hui Za Zhi 38:418-428

19. Berry MN, Friend DS 1969 High-yield preparation of isolated rat liver parenchymal cells: a biochemical and fine structural study. J Cell Biol 43:506-520 
20. Plat J, Brzezinka H, Lutjohann D, Mensink RP, von Bergmann K 2001 Oxidized plant sterols in human serum and lipid infusions as measured by combined gas-liquid chromatography-mass spectrometry. J Lipid Res 42:2030-2038

21. Landrier JF, Eloranta JJ, Vavricka SR, Kullak-Ublick GA 2006 The nuclear receptor for bile acids, FXR, transactivates human organic solute transporter-alpha and -beta genes. Am J Physiol Gastrointest Liver Physiol 290:G476-G485

22. Holt JA, Luo G, Billin AN, Bisi J, McNeill YY, Kozarsky KF, Donahee M, Wang DY, Mansfield TA, Kliewer SA, Goodwin B, Jones SA 2003 Definition of a novel growth factor-dependent signal cascade for the suppression of bile acid biosynthesis. Genes Dev 17:1581-1591

23. Ananthanarayanan M, Balasubramanian N, Makishima M, Mangelsdorf DJ, Suchy FJ 2001 Human bile salt export pump promoter is transactivated by the farnesoid X receptor/bile acid receptor. J Biol Chem 276:28857-28865

24. Colomb V, Jobert-Giraud A, Lacaille F, Goulet O, Fournet JC, Ricour C 2000 Role of lipid emulsions in cholestasis associated with long-term parenteral nutrition in children. JPEN J Parenter Enteral Nutr 24:345-350

25. Salen G, Horak I, Rothkopf M, Cohen JL, Speck J, Tint GS, Shore V, Dayal B, Chen T, Shefer S 1985 Lethal atherosclerosis associated with abnormal plasma and tissue sterol composition in sitosterolemia with xanthomatosis. J Lipid Res 26:1126-1133

26. Bhattacharyya AK, Connor WE 1974 Beta-sitosterolemia and xanthomatosis. A newly described lipid storage disease in two sisters. J Clin Invest 53:1033-1043

27. Klett EL, Lee MH, Adams DB, Chavin KD, Patel SB 2004 Localization of ABCG5 and ABCG8 proteins in human liver, gall bladder and intestine. BMC Gastroenterol $4: 21$

28. Berge KE, Tian H, Graf GA, Yu L, Grishin NV, Schultz J, Kwiterovich P, Shan B, Barnes R, Hobbs HH 2000 Accumulation of dietary cholesterol in sitosterolemia caused by mutations in adjacent ABC transporters. Science 290:1771-1775

29. Miettinen TA, Klett EL, Gylling H, Isoniemi H, Patel SB 2006 Liver transplantation in a patient with sitosterolemia and cirrhosis. Gastroenterology 130:542-547

30. Tomer G, Ananthanarayanan M, Weymann A, Balasubramanian N, Suchy FJ 2003 Differential developmental regulation of rat liver canalicular membrane transporters Bsep and Mrp2. Pediatr Res 53:288-294
31. Kearns GL, Abdel-Rahman SM, Alander SW, Blowey DL, Leeder JS, Kauffman RE 2003 Developmental pharmacology-drug disposition, action, and therapy in infants and children. N Engl J Med 349:1157-1167

32. Chen HL, Chen HL, Liu YJ, Feng CH, Wu CY, Shyu MK, Yuan RH, Chang MH 2005 Developmental expression of canalicular transporter genes in human liver. J Hepatol 43:472-477

33. Moseley RH, Wang W, Takeda H, Lown K, Shick L, Ananthanarayanan M, Suchy FJ 1996 Effect of endotoxin on bile acid transport in rat liver: a potential model for sepsis-associated cholestasis. Am J Physiol 271:G137-G146

34. Ghose R, Zimmerman TL, Thevananther S, Karpen SJ 2004 Endotoxin leads to rapid subcellular re-localization of hepatic RXRalpha: a novel mechanism for reduced hepatic gene expression in inflammation. Nucl Recept 2:4

35. Zimmerman TL, Thevananther S, Ghose R, Burns AR, Karpen SJ 2006 Nuclear export of retinoid $\mathrm{X}$ receptor $\{\mathrm{alpha}\}$ in response to interleukin-1beta-mediated cell signaling: roles for JNK and SER260. J Biol Chem 281:15434-15440

36. van Mil SW, van der Woerd WL, van der Brugge G, Sturm E, Jansen PL, Bull LN, van den Berg IE, Berger R, Houwen RH, Klomp LW 2004 Benign recurrent intrahepatic cholestasis type 2 is caused by mutations in ABCB11. Gastroenterology 127:379-384

37. Nishimura T, Utsunomiya Y, Hoshikawa M, Ohuchi H, Itoh N 1999 Structure and expression of a novel human FGF, FGF-19, expressed in the fetal brain. Biochim Biophys Acta 1444:148-151

38. Inagaki T, Choi M, Moschetta A, Peng L, Cummins CL, McDonald JG, Luo G, Jones SA, Goodwin B, Richardson JA, Gerard RD, Repa JJ, Mangelsdorf DJ, Kliewer SA 2005 Fibroblast growth factor 15 functions as an enterohepatic signal to regulate bile acid homeostasis. Cell Metab 2:217-225

39. Xie W, Radominska-Pandya A, Shi Y, Simon CM, Nelson MC, Ong ES, Waxman DJ, Evans RM 2001 An essential role for nuclear receptors SXR/PXR in detoxification of cholestatic bile acids. Proc Natl Acad Sci U S A 98:3375-3380

40. Shih DM, Kast-Woelbern HR, Wong J, Xia YR, Edwards PA, Lusis AJ 2006 A role for FXR and human FGF-19 in the repression of paraoxonase-1 gene expression by bile acids. J Lipid Res 47:384-392 